\title{
Lipid Peroxidation and Membrane Aging
}

\author{
BERNARD W. AGRANOFF \\ The University of Michigan, Neuroscience Laboratory Building, Ann Arbor, MI 48109
}

\begin{abstract}
This critique points out that a number of possible alterations in cell metabolism could lead to changes in membrane structure and that the observed effects might not necessarily play a primary role in the physiology of aging. Experimental approaches, while not simple, should distinguish causes of altered membrane asymmetry from its consequences.
\end{abstract}

THE idea that cross-linking agents might play a role in the aging process arose initially from studies on the tanning of animal hides and later from research on the synthesis of organoplastics [1]. That malondialdehyde could be the cross-linking agent produced in vivo from the peroxidation of polyunsaturated fatty acids also came first from industry, as a result of investigations on the rancidity of foodstuffs. The theory proposed by Schroeder [8] states further that the cross-linking somehow leads to loss of membrane asymmetry and that this deficit accounts for the pathophysiological changes seen in aging. In regard to the possibility that malondialdehyde is an endogenous cross-linker, additional support comes from studies indicating that temporal changes in erythrocyte membranes may be accounted for by malondialdehyde production [4]. Also, the detection of ethane and pentane in the breath are indications of the in vivo peroxidation of $\omega 3$ and $\omega 6$ fatty acids, respectively [7,9], and will also result in malondialdehyde production. Nevertheless, the contribution of other products of lipid peroxidation to the initiation of degenerative processes should be considered. For example, free radicals, which could lead to tissue damage, are generated. Also, the lysophospholipids produced following fatty acid release may also have detrimental effects. Oxidation or peroxidation products of other lipids such as the terpenoids, should additionally be considered. Batten's disease is often referred to as a model for aging of the nervous system, since lipofuscin is produced. In this instance, altered dolichol metabolism has been reported [5]. A peroxidase defect has also been described in this disease, but the claim is at present controversial. Oxidized cholesterol may also have deleterious effect on membranes. The presence of 25-hydroxycholesterol in the diet has been reported to produce vascular damage [6]. If malondialdehyde indeed proves to be the culprit, Schroeder's theory would be sharpened by a proposal for precisely how it produces its damage. While it can cross-link amino groups of phosphatidylethanolamine and phosphatidylserine, malondialdehyde can also cross-link proteins. The cross-linking of such important proteins as ankyrin or spectrin (fodrin in the nervous system) would seem to suffice to limit membrane mobility without invoking additional primary effects.

While one can readily accept the notion that altered methylation of phospholipids, the loss of diacylglycerol (DG) or a change in cholesterol content can impair membrane asymmetry [8], it is not apparent how these alterations could result from peroxidation. Thus, even if the two parts of the theory, namely the role of peroxidation and malondialdehyde production on the one hand and loss of membrane asymmetry on the other, are both proven to be valid, one needs in addition to prove causality. The proposal that peroxidation leads to lower DG and thus slows phosphatidylcholine and phosphatidylethanolamine synthesis needs further clarification. Newly synthesized phospholipids contain very little unsaturation; polyunsaturated fatty acids that eventually appear in the $s n-2$ position of these phospholipids are generally believed to be added by subsequent deacylation-reacylation reactions catalyzed by phospholipase $A_{2}$ and acyl CoA transferase, respectively. An exception may be found in the inositides, in which a fraction of a specific DG (1-stearoyl, 2-arachidonoyl-sn-glycerol) appears to be recycled [2]. The demonstration of DG as the modulator of a protein kinase suggests a possible regulatory role for this lipid, and it is therefore important to establish whether or not membrane DG is indeed reduced with age.

The erythrocyte membrane has proved to be an excellent model for the study of membrane asymmetry, and it may be suitable for the study of membrane aging as well. It is interesting that erythrocyte membranes contain polyphosphoinositides, as well as a phosphoinositide phospholipase C. This enzyme has been implicated in cellular messenger transduction [2], yet to our knowledge the red cell does not contain relevant membrane receptors. The presence of these lipids and enzymes may be explained by the proposal that the inositides (which appear to be confined to the inner leaflet) play an important role in the maintenance of membrane asymmetry [3]. It has also been proposed that ATP is required to drive phosphatidylethanolamine into the inner leaflet and to maintain its asymmetrical distribution [3]. Thus, decreases in cellular ATP for any of a number of reasons could also lead to the loss of membrane asymmetry.

It would appear that even the simplest of membranes is extremely complex, and that a number of alterations could lead to loss of membrane asymmetry. Proof of the theory would require demonstration that the production of membrane asymmetry is causal rather than a concomitant of some other change in the physiological status of the cell. At present, the number of possibilities are too great for one to propose a simple experiment that could prove or disprove the theory.

The nerve cell differs from the erythrocyte in many ways. It generally survives for the life of the organism, even though its membrane is undergoing dynamic turnover. In the nerve 
cell, the possible loss of a preexisting repair process as a result of age is thus appealing, since accumulations over a long period of time are possible. The brain is an excellent candidate for the ravages of peroxidation, both because of its highly active oxidative metabolism, and because it contains the most polyunsaturated fatty acids in the body. The inositides are enriched in 20:4 66 (arachidonate), but do not constitute a high percentage of total brain phospholipids. The major brain phospholipid is phosphatidylethanolamine. In gray matter, phosphatidylethanolamine contains large amounts of $22: 5 \omega 6$ and $22: 6 \omega 3$ fatty acids. It is of additional interest that 20:5 3 is enriched in fish oils, the administration of which have recently been considered for the prevention of stroke and other thromboembolic disease. If these latter polyunsaturated acids are incorporated into membranes, they could prove to be additional substrates for peroxida- tion, and potentially could lead to increased rates of membrane aging. A possible increased dietary requirement for antioxidants when fish oils are administered should therefore be considered.

More issues have been raised than have been answered by this proposal that lipid peroxidation leads to a loss of membrane asymmetry, and that the loss of asymmetry accounts for the aging of membranes. We can be encouraged, nevertheless, that progress currently being made on our understanding of factors leading to membrane asymmetry will eventually prove useful. It is hoped that the consideration of these issues will lead to experimentation which will further delimit critical parameters which must ultimately be defined in relating age to the loss of structural and functional integrity of cell membranes.

\title{
REFERENCES
}

1. Bjorksten, J. The cross-linkage theory of aging. J Am Geriatr SOC 16: 408-427, 1968.

2. Fisher, S. K., L. A. A. Van Rooijen and B. W. Agranoff. Renewed interest in the polyphosphoinositides. Trends Biochem Sci 9: 53-56, 1984.

3. Gratzer, W. Cell biology: more red than dead. Nature 310: 368$369,1984$.

4. Hochstein, P. and S. K. Fain. Association of lipid peroxidation and polymerization of membrane proteins with erythrocyte aging. Fed Proc 40: 183-188, 1981.

5. Ng Ying Kin, N. M. K., J. Palo, M. Haltia and L. S. Wolfe. High levels of brain dolichols in neuronal ceroid-lipofuscinosis and senescence. $J$ Neurochem 40: 1465-1473, 1983.
6. Peng, S. K. and C. B. Taylor. Atherogenic effect of oxidized cholesterol. In: Dietary Fats and Health, edited by E. G. Perkins and W. J. Visek. Champaign, IL: American Oil Chemists' Society, 1983 , p. 919 .

7. Riely, C. A., G. Cohen and M. Lieberman. Ethane evolution: a new index of lipid peroxidation. Science 183: 208-210, 1974.

8. Schroeder, F. Role of membrane lipid asymmetry in aging. Neurobiol Aging 5: 000-000, 1984.

9. Tappel, A. L. and D. J. Dillard. In vivo lipid peroxidation: measurement via exhaled pentane and protection by vitamin E. Fed Proc 40: 174-178, 1981.

\section{Membrane Lipids and Aging}

\author{
PAUL DEMEDIUK AND LLOYD A. HORROCKS \\ Department of Physiological Chemistry, The Ohio State University \\ 214 Hamilton Hall, 1645 Neil Avenue, Columbus, OH 43210
}

\begin{abstract}
The concept that age-related changes in lipids of brain membranes can "damage" membranes through the loss of communicative coupling of bilayer halves of biomembranes is critiqued and expanded to include the possibility that changes in the asymmetrically distributed gangliosides, in ethanolamine plasmalogens, and in cholesterol may also contribute to such damage. The possibility for attenuation or reversal of lipid changes by dietary manipulations or treatment with liposomes composed of phosphatidylserine is also briefly introduced.
\end{abstract}

THE hypothesis that detrimental changes in the overall composition of membrane lipids occur with both normal and pathological aging has received increasing attention in the past decade. Investigations of this postulate have ranged from simple documentation of the types of lipid changes which occur with aging $[18,27]$ to attempts to correlate such changes with alterations in the structure and dynamics of biological membrones [11, 28, 34]. Many of these studies have discussed the effects of the observed changes in the context of a generic, relatively homogeneous, fluid-mosaic type [31] of biomembrane. The functional implications of such lipid variations have derived from observations of model and biological membranes. These demonstrate that compositional changes such as alterations in length and sat- 